\title{
EPIDEMIOLOGICAL AND PATHOLOGICAL FEATURES OF PRIMARY CARDIAC TUMOURS IN DOGS FROM POLAND IN 1970-2014
}

\author{
Izabela JANUS $^{1 *}$, Marcin NOWAK ${ }^{1}$, Agnieszka NOSZCZYK-NOWAK ${ }^{2}$, Rafał CIAPUTA ${ }^{1}$, \\ Małgorzata KANDEFER-GOLA ${ }^{1}$, Urszula PASŁAWSKA ${ }^{2}$, Rafał SAPIERZYŃSKII ${ }^{3}$, \\ Wojciech ŁOPUSZYŃSKI ${ }^{4}$ and Iwona OTROCKA-DOMAGAŁA ${ }^{5}$
}

${ }^{1}$ Department of Pathology and ${ }^{2}$ Department of Internal Medicine and Clinic of Diseases of Horses, Dogs and Cats, Wroclaw University of Environmental and Life Sciences,

C. K. Norwida 31, 50375 Wroclaw, Poland; ${ }^{3}$ Department of Pathology and Veterinary Diagnostics, Faculty of Veterinary Medicine, Warsaw University of Life Sciences (SGGW), Warsaw, Poland; ${ }^{4}$ Department of Pathological Anatomy, Faculty of Veterinary Medicine, University of Life Sciences, Lublin, Poland; ${ }^{5}$ Department of Pathological Anatomy, Warmia and Mazury University in Olsztyn, Olsztyn, Poland

(Received 26 May 2015; accepted 28 October 2015)

Primary heart tumours affect less than $1 \%$ of dogs. Due to their rare incidence, every research showing the frequency of cardiac tumours is valuable. Routine diagnostics is often complemented with immunohistochemical analysis. This study was conducted on 110 patient records from all veterinary faculties in Poland from dogs diagnosed with heart tumours between 1970 and 2014. The dogs' age, breed and sex with tumour localisation and histopathological diagnosis were analysed. Because of its most common incidence, samples of haemangiosarcoma underwent further examination with assessment of the expression of cell markers that have not been evaluated earlier (i.e. minichromosome maintenance proteins and beta-catenin). We noted 111 tumours including 88.3\% malignant and 10.8\% benign ones. Haemangiosarcoma and aortic body tumour were the most frequent cardiac neoplasms in the dogs examined $(45.9 \%$ and $27.9 \%$ of all tumours, respectively). Immunohistochemical analysis of haemangiosarcoma showed a positive expression of all markers examined. CD31, vimentin, and beta-catenin showed a positive reaction in all 11 samples examined. At least one proliferative marker (Ki-67, MCM-3 or MCM-7) showed a positive reaction in each sample. MCM-3 showed a higher expression than the two other proliferative markers $(\mathrm{P}=$ $0.006)$, but only Ki-67 showed a positive correlation with the mitotic index $(\mathrm{P}<$ $0.05, \mathrm{r}=0.89)$. Although beta-catenin, MCM-3 and MCM-7 showed a positive reaction in the haemangiosarcomas examined, their usefulness as diagnostic and prognostic factors should be a topic of further research.

Key words: Cardiac tumour, haemangiosarcoma, immunohistochemistry, $\operatorname{dog}$

*Corresponding author; E-mail: izabela.janus87@gmail.com; Phone/Fax: 0048 (71) 320-5415 
Primary heart tumours are a rare cardiac condition affecting less than $1 \%$ of humans and animals (Lam et al., 1993; Ware and Hopper, 1999; Agaimy et al., 2012). Regardless of their rare occurrence, cardiac tumours often cause severe clinical symptoms including signs of heart failure, arrhythmias, sudden death, or non-specific signs such as anorexia or apathy (Ware and Hopper, 1999; Aupperle et al., 2007; Rajagopalan et al., 2013). The clinical examinations used for the diagnosis of cardiac tumours (including echocardiography, pericardiocentesis, and tumour biopsy) are often characterised by insufficient accuracy or high risk (Rajagopalan et al., 2013). Only the histopathological examination of heart tumour specimens is suitable for the precise recognition of cardiac neoplasms.

The published frequency of different cardiac neoplasms depends on the examined population but there is agreement in the literature that in dogs malignant tumours are more common than benign neoplasms, with haemangiosarcoma (HSA) being the most frequent (Ware and Hopper, 1999; Aupperle et al., 2007). HSA, as a prevalent tumour both in humans and in dogs, was examined for the expression of several cell markers including but not restricted to CD31, FVIIIRA, Ki-67, p53 protein, vimentin, SMA, desmin, S-100 protein, actin, and CD117 (Ferrer et al., 1995; Bertazzolo et al., 2005; Kim et al., 2008; Sabattini and Bettini, 2009; Ge et al., 2011).

Both CD31 and FVIIIRA are specific for normal and neoplastic endothelial cells, yet they do not allow for discrimination between cells originating from lymphatic and blood vessels. Simultaneously, the expression of CD31, as compared to FVIIIRA, is stronger and less diffuse, which suggests a higher diagnostic value of the first-mentioned protein (Ferrer et al., 1995; Bertazzolo et al., 2005).

Vimentin is a component of the cytoskeleton of mesenchymal cells, showing an expression both in normal and neoplastic endothelial cells (Sabattini and Bettini, 2009).

$\mathrm{Ki}-67$ is a cell proliferation marker that is expressed in all phases of the cell cycle except $\mathrm{G}_{0}$ and is routinely used as a diagnostic tool (Gambichler et al., 2009; Kreuter et al., 2009).

Due to the lack of information about the expression of beta-catenin and Mini-Chromosome Maintenance (MCM) proteins in canine HSA, we expanded the examination protocol of that neoplasm with the aforementioned proteins. Those markers have been examined in recent years on various neoplasms to determine their usefulness as diagnostic and prognostic factors (Nowak et al., 2007a,b; Gambichler et al., 2009; Kreuter et al., 2009; Lau et al., 2010; Saydam et al., 2010; Stein et al., 2011).

Beta-catenin is an intercellular protein responsible for two important phenomena: intercellular adhesion (together with cadherins) and transfer of extracellular-initiated Wnt signals to the cell nucleus. The Wnt signalling pathway is connected among others with cell proliferation and differentiation (Nowak et al., 2007a; Stein et al., 2011). In normal cells, beta-catenin expression is limited to 
the cell membrane, while in neoplastic cells it accumulates in the cytoplasm and, to a lesser extent, in the cell nucleus. Changes in the distribution of beta-catenin associated with alterations in the cadherin-catenin complex result in disorders in cell adhesion (cells are released more easily from the primary tumour to form metastases), limitation of cell differentiation, and increased tumour invasiveness. Moreover, the accumulation of beta-catenin in the cell nucleus may lead to alterations in the cell cycle and, in consequence, to more intense proliferation and neoplastic transformation (Nowak et al., 2007a,b; Stein et al., 2011).

The MCM proteins 2-7 function as licensing components for the S-phase of the cell cycle. They are proliferative markers like Ki-67 (Gambichler et al., 2009; Kreuter et al., 2009; Lau et al., 2010).

Each new marker helps us understand the pathogenesis of a tumour better and improve the diagnostic and therapeutic possibilities.

The aims of this study were to determine (1) the epidemiology of cardiac tumours in dogs from Poland and (2) the expression of selected cell markers in HSA as the most frequent cardiac neoplasm in dogs.

\section{Materials and methods}

The research was done retrospectively on the basis of gross and histopathological examination records. Specimens from 110 dogs with end-stage neoplastic disease, either collected during routine necropsy or received from private clinics, were subjected to pathological examination during the 44-year period between 1970 and 2014 at all veterinary faculties in Poland.

For histopathological analysis, the sections were fixed in $7 \%$ buffered formalin, dehydrated, and embedded in paraffin blocks. They were stained with haematoxylin and eosin (HE). If needed, immunohistochemical techniques were applied to complete the diagnosis. The dogs' age, sex, breed, tumour localisation, malignancy, and histological type served for classification.

Because of the most common incidence of haemangiosarcoma, paraffin blocks of 11 samples of HSA from the last two years underwent additional immunohistochemical analysis including - apart from the routinely used CD31, vimentin and Ki-67 - beta-catenin, MCM-3 and MCM-7 expression. No other types of cardiac tumours were presented with a sufficient number of samples in the last two years to provide reliable comparisons by expanded immunohistochemical analysis. Specimens older than two years were excluded from extended immunohistochemical analysis due to the possibility of false negative results. The analysis was performed on $4-\mu \mathrm{m}$-thick paraffin sections placed on silanised microscope slides (DAKO, Glostrup, Denmark) using a routine procedure. Sections were overlaid with primary antibodies including Mouse Monoclonal AntiHuman Ki-67 - clone MIB-1 (DAKO, Denmark), diluted 1:50; Rabbit Mono- 
clonal Anti-Human MCM-3 - clone EP202 (BioSB, USA), diluted 1:50; Mouse Monoclonal Anti-Human MCM-7 - clone 101 (DAKO, Denmark), diluted 1:50; Mouse Monoclonal Anti-Human Beta-Catenin - clone $\beta$-Catenin-1 (DAKO, Denmark), diluted 1:100; Mouse Monoclonal Anti-Vimentin - clone V9 (DAKO, Denmark), diluted 1:50; Mouse Monoclonal Anti-Human CD31, Endothelial Cell - clone JC70A, diluted 1:100; all incubated for $30 \mathrm{~min}$ at room temperature. Sections were counterstained with haematoxylin (Sigma-Aldrich, St. Louis, MO, USA). Normal heart specimens stained according to the above-mentioned protocol served as positive controls; sections immunostained in the absence of a primary antibody were used as negative controls.

Photomicrographs of the tumours examined were subjected to computerassisted image analysis, using a computer coupled to an Olympus BX53 optical microscope equipped with an Olympus Color View IIIa digital camera (Olympus, Japan). The analysis was performed with $\mathrm{Cell}^{\wedge} \mathrm{A}$ software (Olympus Soft Imaging Solution GmbH, Germany).

The expression of CD31, vimentin and $\beta$-catenin was appraised using the modified semiquantitative IRS scale according to Remmele and Stegner (1987). The expression of Ki-67, MCM-3 and MCM-7 was evaluated quantitatively by estimation of the percentage of neoplastic cells showing positive reaction $[0-5 \%=$ no reaction $(-), 6-25 \%=$ weak reaction $(+), 26-50 \%=$ moderate reaction $(++)$, above $50 \%=$ intense reaction $(+++)]$. Expression of the markers examined was evaluated in 20 randomly chosen view fields per slide, and an average value for each slide was calculated.

The data were subjected to statistical analysis with Statistica PL for Windows 10.0 software using tests including Spearman's correlation test, MannWhitney U analysis, Friedman's analysis of variance (ANOVA), Kruskal-Wallis analysis, and chi-square analysis. Statistical significance was set at $\mathrm{P} \leq 0.05$.

\section{Results}

In the period 1970 through 2014, a total of 111 cardiac neoplasms were examined (including two tumours from one dog). The number of diagnosed tumours increased with time (Fig. 1).

Among the cases studied, 12/111 (10.8\%) benign and 98/111 (88.3\%) malignant tumours were determined. In one case it was impossible to establish an accurate histopathological diagnosis.

The benign neoplasms included seven fibromas $(6.3 \%$ of all tumours, $58.3 \%$ of benign tumours), four myxomas (3.6\% of all tumours, $33.3 \%$ of benign tumours), and one case of rhabdomyoma ( $0.1 \%$ of all tumours, $8.3 \%$ of benign tumours) (Fig. 2). 


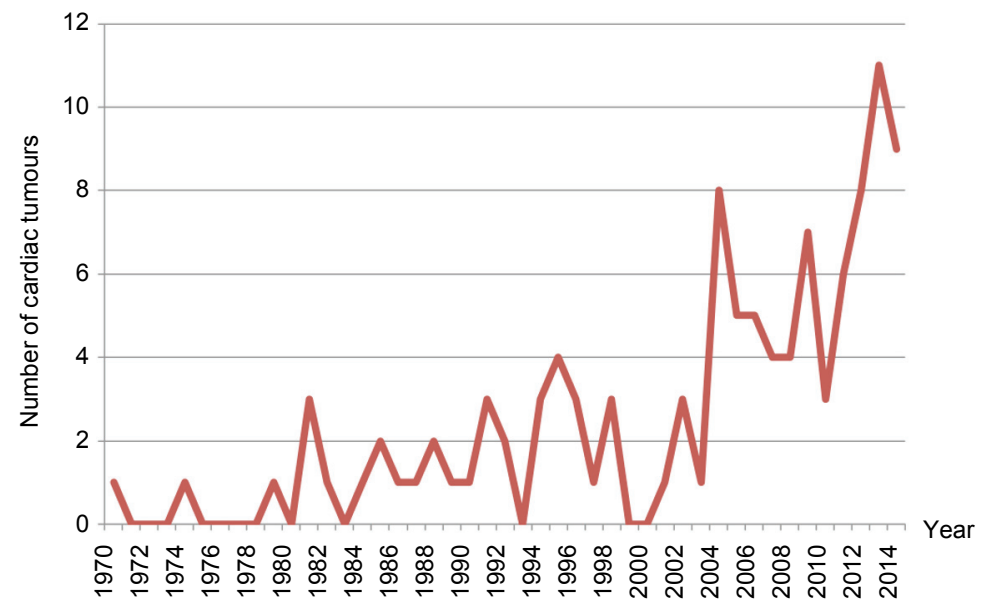

Fig. 1. Number of cardiac tumours diagnosed by year between 1970 and 2014

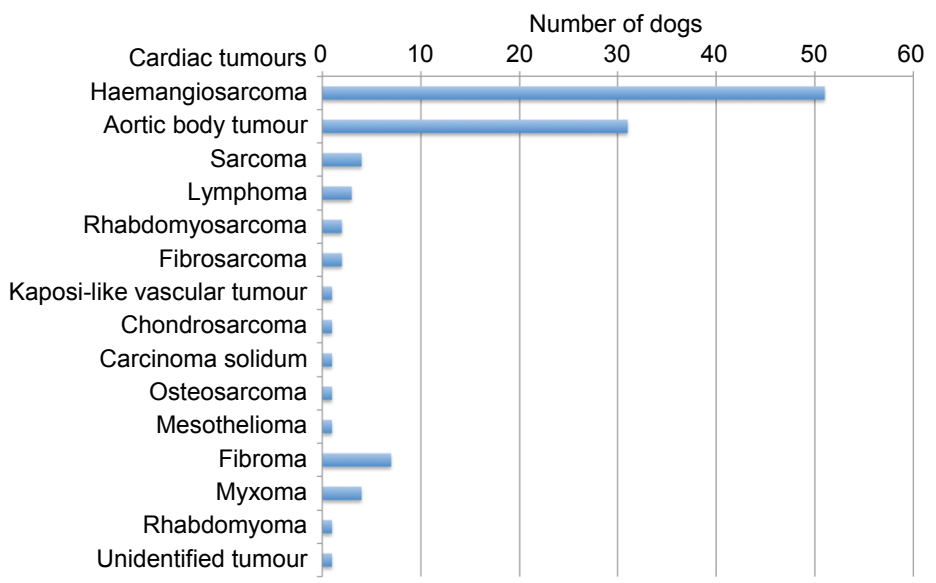

Fig. 2. Frequency of cardiac tumours diagnosed in the dogs examined $(\mathrm{n}=111)$

The malignant neoplasms included 51 HSA ( $45.9 \%$ of all tumours, $52 \%$ of malignant tumours, including 14 cases in German Shepherds), 31 aortic body tumours (ABT, $27.9 \%$ of all tumours, $31.6 \%$ of malignant tumours, including 13 cases in Boxer dogs), four non-differentiated sarcomas $(3.6 \%$ of all tumours, $4.1 \%$ of malignant tumours), and three or less cases of other tumours (Fig. 2).

Due to the retrospective character of the study, in some of the animals it was impossible to determine all of the data. In 47/111 tumours (42.7\%) an accurate tumour localisation was lacking, with only 'heart tumour' or 'cardiac muscle tumour' existing as a description. Among the 51 HSAs diagnosed, in 24 cases $(47.1 \%)$ the localisation was determined as 'atria' or 'atrial auricle', and in five 
cases $(9.8 \%)$ as 'the heart base'. Among the 31 ABTs diagnosed, 13 tumours $(41.9 \%)$ were localised at the heart base. Moreover, in the group of 18 tumours localised at the heart base, all neoplasms were either ABT $(n=13)$ or HSA $(n=5)$, and in the group of 33 tumours localised within the atria (including the atrial auricles), 25 tumours were HSA, two were ABT and one case each was Kaposilike vascular tumour, sarcoma, solid carcinoma, fibroma, myxoma, and a tumour without precise histopathological diagnosis, respectively.

Thirty dogs (27.27\%) were crossbreeds. Among the purebred dogs $(\mathrm{n}=$ $80)$, German Shepherds $(20 \% ; \mathrm{n}=22)$ and Boxers $(16.36 \% ; \mathrm{n}=18)$ were the most common, followed by Dachshunds $(3.64 \% ; n=4)$, while other breeds were represented by three or less dogs (Fig. 3).

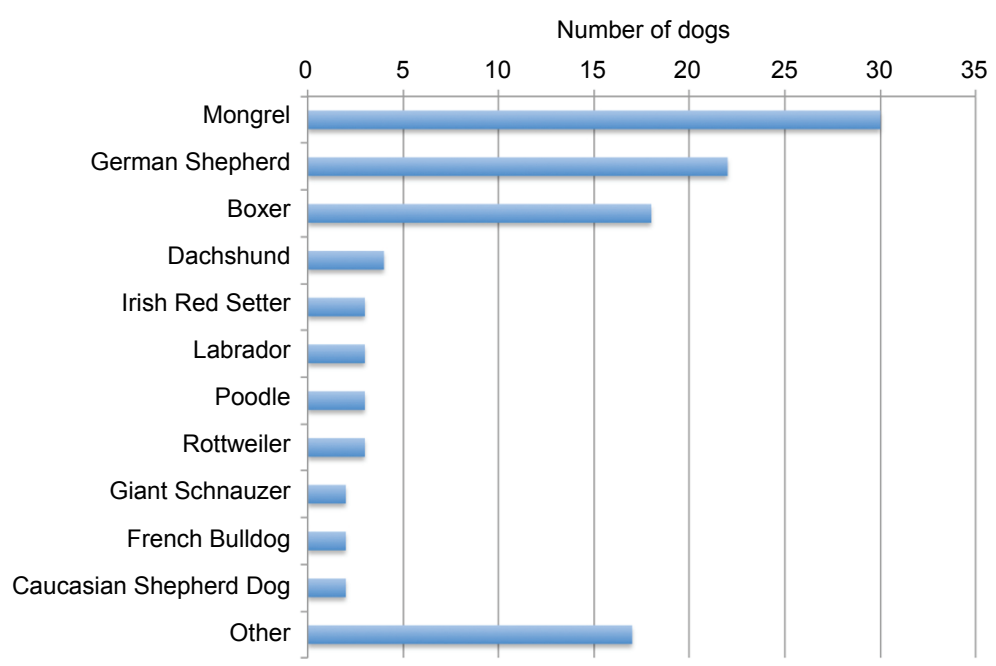

Fig. 3. Breed prevalence of cardiac tumours in dogs $(\mathrm{n}=109)$. 'Other' includes breeds represented by one dog: Golden Retriever, Cane Corso, American Staffordshire Terrier, Miniature Schnauzer, Pekingese, Pointer, Fox Terrier, Jagdterrier, Doberman Pinscher, Leonberger, Belgian Shepherd, Pug, Black Russian Terrier, Bouvier des Flandres, Weimaraner, Beagle, Boston Terrier

Tumours were diagnosed in dogs aged 10 months to 18 years (mean 9.14 years, $\mathrm{SD}=2.82)$ with no significant differences between the number of males and females $(\mathrm{P}=0.104$; chi-square analysis $)$. Among dogs presenting with HSA, a significantly higher number of males than females was noted $(P=0.005$; chisquare analysis), but that difference was not observed in the group of German Shepherds with HSA ( $\mathrm{P}=0.109$; chi-square analysis). In dogs presenting with $\mathrm{ABT}$ and in Boxers with $\mathrm{ABT}$ no significant difference in sex distribution was noted $(\mathrm{P}=0.467$ and $\mathrm{P}=0.781$, respectively; chi-square analysis). For other types of tumours the case numbers were not sufficiently high to provide reliable results on sex distribution. 
We found no differences in age between dogs of different sex $(\mathrm{P}=0.969$; Mann-Whitney $\mathrm{U}$ analysis), dogs presenting with different types of tumours ( $\mathrm{P}=$ 0.599; Kruskal-Wallis rank analysis), and dogs presenting with benign and malignant tumours $(\mathrm{P}=0.776$; Mann-Whitney $\mathrm{U}$ analysis).

The immunohistochemical analysis of HSA showed intense $(+++)$ expression of CD31 in all 11 cases $(100 \%)$, which confirmed the endothelial origin of these tumours. Vimentin was expressed moderately $(++)$ in $4 / 11(36.4 \%)$ and intensely (+++) in $7 / 11(63.6 \%)$ cases, and the cytoplasmic expression of betacatenin was noted in all 11 tumours, including a weak $(+)$ reaction in 5/11 cases $(45.5 \%)$, a moderate $(++)$ reaction in $4 / 11$ cases $(36.4 \%)$, and an intense $(+++)$ reaction in $2 / 11$ cases $(18.2 \%)$. In each of the tumours examined at least one proliferation marker (Ki-67, MCM-3, or MCM-7) showed a positive reaction. MCM-3 showed a higher expression than the other two proteins $(\mathrm{P}=0.006$; Friedman's ANOVA). A positive expression of MCM-3 was noted in 10/11 cases, including a weak $(+)$ reaction in $5 / 11$ cases $(45.5 \%)$, a moderate $(++)$ reaction in $4 / 11$ cases $(36.4 \%)$, and an intense $(+++)$ reaction in $1 / 11(9.1 \%)$ case. A positive expression of both Ki-67 and MCM-7 was observed in 8/11 tumours, including a weak $(+)$ reaction in $7 / 11$ cases $(63.6 \%)$ and a moderate $(++)$ reaction in $1 / 11$ case $(9.1 \%)$ (Figs $4-5)$. We observed no correlation in expression between the proteins examined ( $\mathrm{P}>0.05$, Spearman's correlation test). Only Ki-67 showed a high positive correlation with the mitotic index $(\mathrm{P}<0.05, \mathrm{r}=0.89$, Spearman's correlation test).

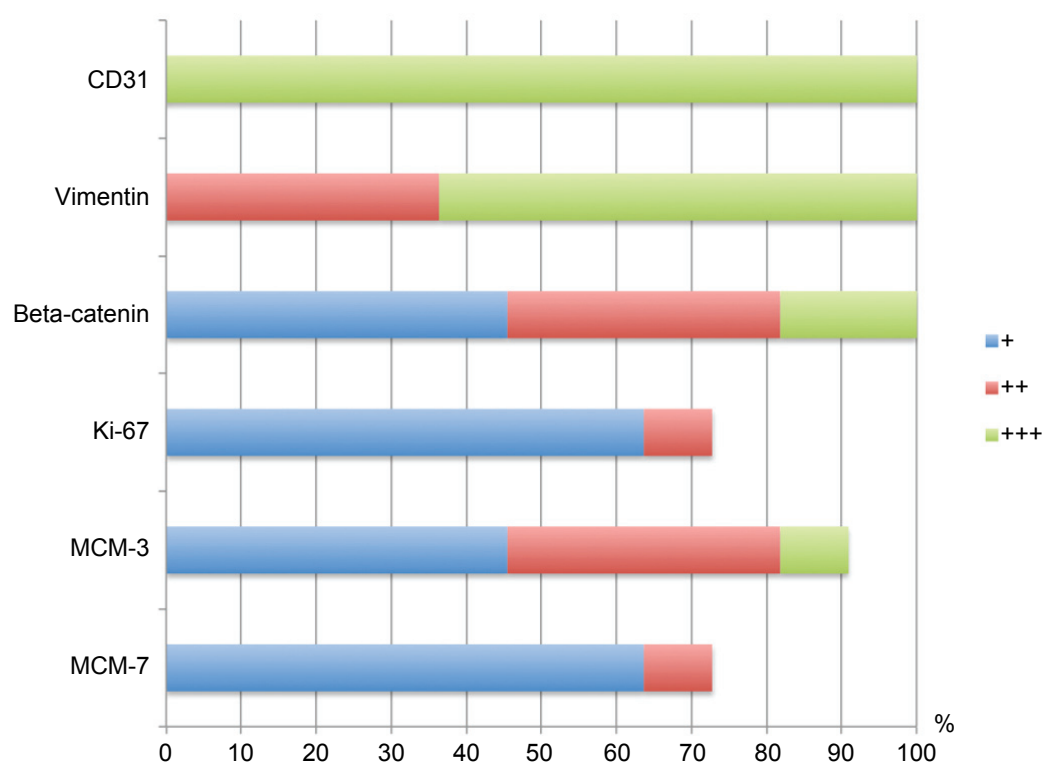

Fig. 4. The percentage of haemangiosarcoma samples showing a positive reaction to the markers examined $(\mathrm{n}=11) ;+=$ weak, $++=$ moderate,$+++=$ intense reaction 


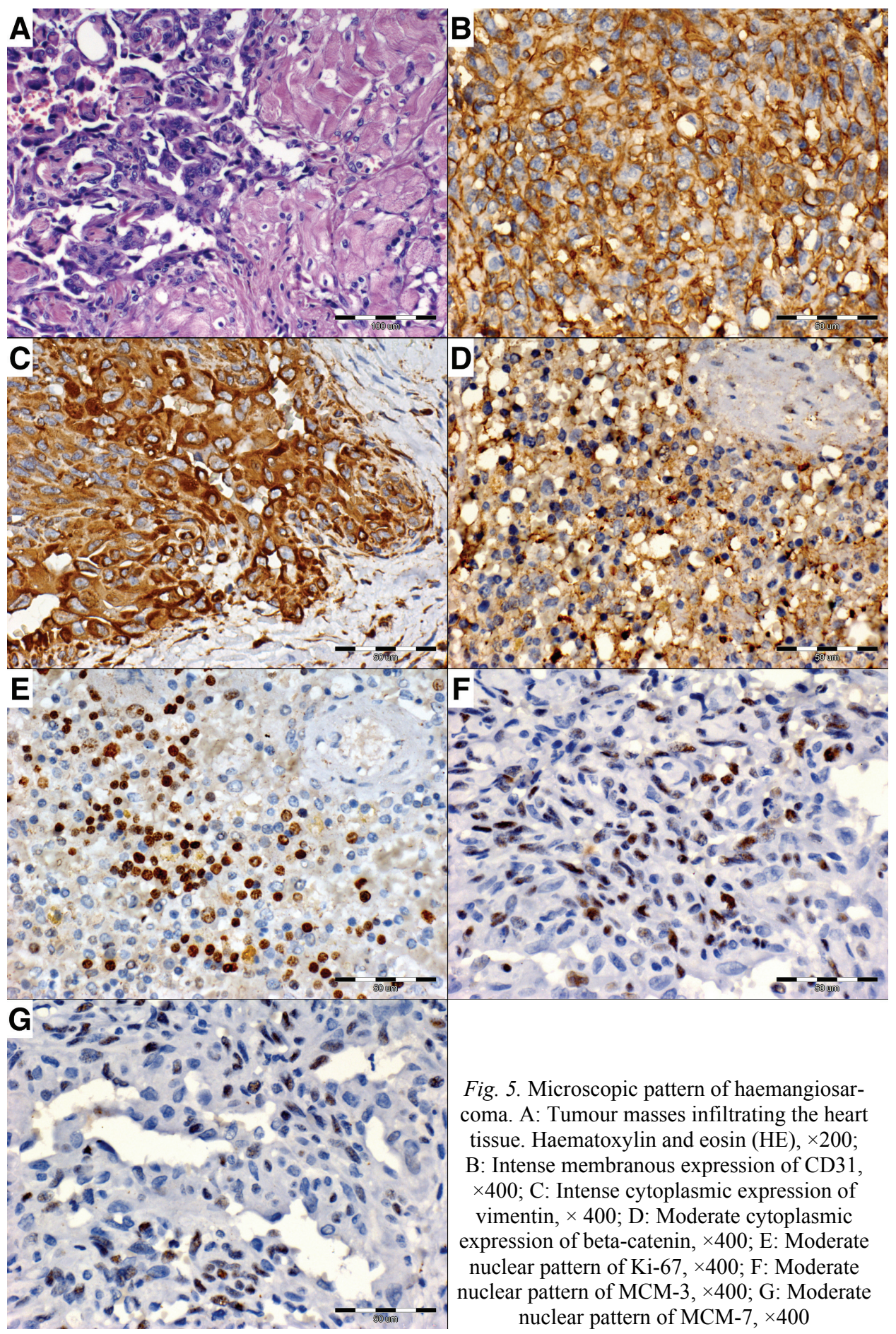




\section{Discussion}

Along with the growing awareness of pet owners and the increasing life expectancy of animals, the number of tumours diagnosed in dogs (including cardiac tumours) steadily rises (Ciaputa et al., 2013; Gesek et al., 2014). Ware and Hopper (1999) and Aupperle et al. (2007) noted primary cardiac neoplasms mainly in middle-aged large breed dogs, without a sex predisposition. Also in our study, 84 dogs $(76.4 \%)$ were aged between 7 and 15 years and only one dog was older than 15 years.

Only in cases of HSA could a distinct sex-related difference be observed (34 males vs. 17 females). Together with the equal male/female ratio in the entire dog cohort examined, and considering the results obtained by Sabattini and Bettini (2009), this finding suggests a predisposition of males to develop cardiac HSA. Other authors (Ware and Hopper, 1999; Aupperle et al., 2007) did not find such a relationship. We observed a trend of HSA to localise within the atria and of ABT to localise at the heart base, which was also confirmed in other studies (Ware and Hopper, 1999; Aupperle et al., 2007; Noszczyk-Nowak et al., 2010). A considerable percentage of both HSA and ABT occurred in other localisations (including the ventricular walls) and a significant number of tumours had nondefined localisations. This finding, together with the results of Rajagopalan et al. (2013) showing a low accuracy of preliminary diagnosis based on tumour localisation, does not allow us to draw far-reaching conclusions on the typical localisation of the tumours mentioned above.

A high percentage of crossbreeds among all dogs and of German Shepherds and Boxers among purebred dogs, similar to that reported by Ciaputa et al. (2013) and Gesek et al. (2014), may be related to the popularity of these dogs in Poland, rather than to their predisposition to develop neoplastic lesions. On the other hand, HSA occurring in 14 out of 22 German Shepherds and ABT in 13 out of 18 Boxers in our study, together with the results obtained by other authors (Brown et al., 1985; Ware and Hopper, 1999; Sabattini and Bettini, 2009), and the low percentage of Dachshunds and the absence of Yorkshire Terriers (breeds also popular in Poland) among dogs affected by these tumours, may confirm the predisposition of the former breeds to develop certain types of tumours. It is suspected that chronic hypoxia occurring in brachycephalic dogs may play a role in the stimulation of neoplastic growth originating from chemoreceptor cells (Noszczyk-Nowak et al., 2010), although not all dogs diagnosed with ABT were brachycephalic either according to the literature (Ware and Hopper, 1999) or in our study.

In humans, benign cardiac tumours are more frequent than malignant ones, with myxomas being the most common benign neoplasms and myxosarcomas, HSA or non-differentiated sarcomas being the most common malignant tumours (Lam et al., 1993; Kim et al., 2008; Kumar et al., 2012; Wang et al., 2012). The 
studies of Ware and Hopper (1999) and Aupperle et al. (2007) show that among tumours diagnosed in dogs post mortem, malignant tumours are more frequent, with HSA and ABT being the most common. A higher incidence of malignant tumours in dogs than in humans may be connected with the predisposition of dogs to developing malignant neoplasms (mainly HSA and ABT).

HSA as a frequent tumour both in humans and animals was evaluated for various cell markers. According to the literature, cardiac HSA in humans shows positive expression of CD31, CD34, FLI-1, Ki-67, FVIIIRA, and p53 protein, and negative expression of SMA, desmin, S-100 protein, cytokeratin, c-kit protein, EGFR, and CD45 (Lam et al., 1993; Ge et al., 2011).

In dogs, immunohistochemical studies of angiosarcoma included the expression of CD31 and FVIIIRA with a stronger and less diffuse reaction of the first marker (Ferrer et al., 1995; Bertazzolo et al., 2005). In the present study we also observed a strong reaction of CD31 in all tumour specimens, which confirms their endothelial origin.

In a study of canine haemangioma and HSA, Sabattini and Bettini (2009) showed in the second group a distinct expression of vimentin, actin, FVIIIRA, CD117, VEGFR-3, and CD44 with a low expression of VEGFC. Similarly as in the above-mentioned research, in our study vimentin showed moderate or intense expression in all specimens examined. The lack of correlation observed between the expression of vimentin and that of proliferative proteins (Ki-67, MCM-3, and MCM-7) confirms the results obtained by Sabattini and Bettini (2009), showing a similar intensity of vimentin expression in both haemangioma and HSA.

Although both Ki-67 and MCM family proteins play a role during cell division and DNA replication, the intensity of their expression in the examined tissues may be diverse (Gambichler et al., 2009; Kreuter et al., 2009; Lau et al., 2010; Saydam et al., 2010). Similarly, in this study the expression of three proliferative proteins showed differences, but each examined tumour exhibited a positive reaction for at least one of the above-mentioned markers. The results of previous studies suggest that the expression of MCM family proteins can be used as a tool in differentiating between normal and neoplastic tissue (Saydam et al., 2010) or between benign and malignant tumours (Gambichler et al., 2009; Kreuter et al., 2009). Lau et al. (2010) noted that high MCM-3 expression correlates with poor prognosis in human melanoblastoma. Although the positive expression of all examined proliferation markers noted in the HSAs examined seems promising, the lack of correlation between MCM family proteins and the mitotic index or Ki-67 may suggest that those markers are not useful in the examination of canine HSA. Further studies with more samples of HSA and haemangioma should be conducted to determine the usefulness of those proteins in the diagnosis of cardiac HSA in dogs.

As mentioned before, the transformation of beta-catenin expression from membrane to cytoplasmic/nuclear is frequent in various canine neoplasms and 
may be related to the neoplastic transformation of tissues (Nowak et al., 2007a,b; Stein et al., 2011). In this study the expression of beta-catenin was mainly cytoplasmic, indicating alterations in cell adhesion. Although in this study, as in the research of Nowak et al. (2007a), no correlation was observed between the expression of beta-catenin and that of proliferative proteins, the findings of other studies (Nowak et al., 2007b) show a positive correlation between those markers and suggest the possible role of beta-catenin in the proliferation of neoplastic cells.

Recent studies show a high diagnostic potential of beta-catenin and MCM family proteins in the histopathological analysis of various malignant neoplasms (Nowak et al., 2007a,b; Gambichler et al., 2009; Kreuter et al., 2009; Lau et al., 2010; Saydam et al., 2010; Stein et al., 2011; Galofaro et al., 2012). Extension of the examination protocol by MCM family proteins allows a more accurate assessment of the proliferative potential of tumours. The analysis of beta-catenin expression with the subsequent examination of disorders in the Wnt/beta-catenin signalling pathway not only permits a better understanding of neoplastic pathogenesis but also should lead to attempts to discover new antineoplastic drugs influencing that pathway (Nowak et al., 2007a).

This study had several limitations. The research was conducted retrospectively on the basis of examination records, which may increase the risk of diagnostic errors in the presented dataset. Information about the clinical signs, the time from initial diagnosis to death or euthanasia, and complete information about metastases could raise the value of the paper. Moreover, because of the relatively small number of HSA samples subjected to immunohistochemical examination, the lack of clinical data about those patients and the absence of haemangioma samples serving for comparison, the conclusions drawn on the use of beta-catenin and MCM family proteins in the diagnostic process should be regarded only as preliminary results and a basis for further research.

In conclusion, it can be established that the growing awareness of pet owners, the longer life expectancy of animals and the improved diagnostic possibilities result in an increasing number of recognised cardiac neoplasms. Our results indicate a breed predisposition for specific tumours, i.e. HSA in German Shepherds and ABT in Boxers.

Based on our results, MCM family proteins and beta-catenin can be used for the diagnosis of HSA in dogs, but this topic requires further, more detailed studies performed on a higher number of cases.

\section{Acknowledgement}

The authors would like to thank Aleksandra Raźniewska, 5th-year student at the Faculty of Veterinary Medicine, Warsaw University of Life Sciences (SGGW), for her help in collecting the cases of cardiac tumours. 


\section{References}

Agaimy, A., Rösch, J., Weyand, M. and Strecker, T. (2012): Primary and metastatic cardiac sarcomas: a 12-year experience at a German heart centre. Int. J. Exp. Pathol. 5, 928-938.

Aupperle, H., März, I., Ellenberger, C., Buschatz, S., Reischauer, A. and Schoon, H.-A. (2007): Primary and secondary heart tumours in dogs and cats. J. Comp. Pathol. 136, 18-26.

Bertazzolo, W., Dell'Orco, M., Bonfanti, U., Ghisleni, G., Caniatti, M., Masserdotti, C., Antoniazzi, E., Crippa, L. and Roccabianca, P. (2005): Canine angiosarcoma: cytologic, histologic, and immunohistochemical correlations. Vet. Clin. Path. 34, 28-34.

Brown, N. O., Patnaik, A. K. and MacEwen, E. G. (1985): Canine hemangiosarcoma: retrospective analysis of 104 cases. JAVMA 186, 56-58.

Ciaputa, R., Kandefer-Gola, M., Nowak, M. and Madej, J. A. (2013): Prevalence of tumours in domestic animals in the Lower Silesia (Poland) in 2009-2011. Bull. Vet. Inst. Pulawy 57, $53-59$.

Ferrer, L., Fondevila, D., Rabanal, R. M. and Vilafranca, M. (1995): Immunohistochemical detection of CD31 antigen in normal and neoplastic canine endothelial cells. J. Comp. Path. 112, 319-326.

Galofaro, V., Rapisarda, G., Sfacteria, A., Lanteri, G. and Marino, F. (2012): Beta-catenin expression in pilomatrix carcinoma with multiple visceral metastases in a dog. Acta Vet. Hung. 60, $449-457$.

Gambichler, T., Shtern, M., Rotterdam, S., Bechara, F. G., Stücker, M., Altmeyer, P. and Kreuter, A. (2009): Minichromosome maintenance proteins are useful adjuncts to differentiate between benign and malignant melanocytic skin lesions. J. Am. Acad. Dermatol. 60, 808-813.

Ge, Y., Ro, J. Y., Kim, D., Kim, C. H., Reardon, M. J., Blackmon, S., Zhai, J., Coffey, D., Benjamin, R. S. and Ayala, A. G. (2011): Clinicopathologic and immunohistochemical characteristics of adult primary cardiac angiosarcomas: analysis of 10 cases. Ann. Diagn. Pathol. 15, 262-267.

Gesek, M., Rotkiewicz, T., Otrocka-Domagała, I., Paździor-Czapula, K., Kozłowska, A., Korzeniowska, P., Grochowska, K. and Welenc, J. (2014): Manifestation of tumours in domestic animals in Warmia and Mazury (Poland) between 2003 and 2011. Bull. Vet. Inst. Pulawy 58, 439-446.

Kim, C. H., Dancer, J. Y., Coffey, D., Zhai, Q. J., Reardon, M., Ayala, A. and Ro, J. Y. (2008): Clinicopathologic study of 24 patients with primary cardiac sarcomas: a 10-year single institution experience. Hum. Pathol. 39, 933-938.

Kumar, N., Agarwal, S., Ahuja, A., Das, P., Airon, B. and Ray, R. (2012): Spectrum of cardiac tumors excluding myxoma: experience of a tertiary center with review of the literature. Pathol. Res. Pract. 207, 769-774.

Kreuter, A., Jesse, M., Potthoff, A., Brockmeyer, N. H., Gambichler, T., Stücker, M., Bechara, F. G., Pfister, H. and Wieland, U. (2009): Expression of proliferative biomarkers in anal intraepithelial neoplasia of HIV-positive men. J. Am. Acad. Dermatol. 63, 490-498.

Lam, K. Y., Dickens, P. and Chan, A. C. (1993): Tumors of the heart. A 20-year experience with a review of 12,485 consecutive autopsies. Arch. Pathol. Lab. Med. 117, 1027-1031.

Lau, K. M., Chan, Q. K., Pang, J. C., Li, K. K., Yeung, W. W., Chung, N. Y., Lui, P. C., Tam, Y. S., Li, H. M., Zhou, L., Wang, Y., Mao, Y. and Ng, H. K. (2010): Minichromosome maintenance proteins 2, 3 and 7 in medulloblastoma: overexpression and involvement in regulation of cell migration and invasion. Oncogene 29, 5475-5489.

Noszczyk-Nowak, A., Nowak, M., Pasławska, U., Atamaniuk, W. and Nicpon, J. (2010): Cases with manifestation of chemodectoma diagnosed in dogs in Department of Internal Diseases with Horses, Dog and Cat Clinic, Veterinary Medicine Faculty, University of Environmental and Life Sciences, Wroclaw, Poland. Acta Vet. Scand. 52, 35. 
Nowak, M., Madej, J. A. and Dzięgiel, P. (2007a): Expression of E-cadherin, $\beta$-catenin and Ki-67 antigen and their reciprocal correlations in fibrosarcomas of soft tissues in dogs. In Vivo 21, 751-756.

Nowak, M., Madej, J. A. and Dzięgiel, P. (2007b): Expression of E-cadherin, $\beta$-catenin and Ki-67 antigen and their reciprocal relationships in mammary adenocarcinomas in bitches. Folia Histochem. Cytobiol. 45, 233-238.

Rajagopalan, V., Jesty, S. A., Craig, L. E. and Gompf, R. (2013): Comparison of presumptive echocardiographic and definitive diagnoses of cardiac tumors in dogs. J. Vet. Intern. Med. 27, 1092-1096.

Remmele, W. and Stegner, H. E. (1987): Recommendation for uniform definition of an immunoreactive score (IRS) for immunohistochemical estrogen receptor detection (ER-ICA) in breast cancer tissue. Pathologe 8, 138-140.

Sabattini, S. and Bettini, G. (2009): An immunohistochemical analysis of canine haemangioma and haemangiosarcoma. J. Comp. Pathol. 140, 158-168.

Saydam, O., Senol, O., Schaaij-Visser, T. B. M., Pham, T. V., Piersma, S. R., StemmerRachaminov, A. O., Wurdinger, T., Peerdeman, S. M. and Jimenez, C. R. (2010): Comparative protein profiling reveals minichromosome maintenance (MCM) proteins as novel potential tumor markers for meningiomas. J. Proteome Res. 9, 485-494.

Stein, T. J., Holmes, K. E., Muthuswamy, A., Thompson, V. and Huelsmeyer, M. K. (2011): Characterization of $\beta$-catenin expression in canine osteosarcoma. Vet. Comp. Oncol. 9, 65-73.

Wang, J. G., Liu, H., Yu, W. J., Li, Y. J. and Xin, F. J. (2012): Primary cardiac neoplasms: a clinicopathologic analysis of 81 cases. Zhonghua Bing Li Xue Za Zhi [Chinese J. Pathol.] 41, $808-812$.

Ware, W. A. and Hopper, D. L. (1999): Cardiac tumors in dogs: 1982-1995. J. Vet. Intern. Med. 13, 95-103. 